\title{
A Comparative Study of the Numerical Approximations of the Quenching Time for a Nonlinear Reaction-Diffusion Equation
}

\author{
Frederick Jones ${ }^{1}$ and He Yang ${ }^{1 *}$ \\ ${ }^{1}$ Department of Mathematics, Augusta University, Augusta, USA \\ * Corresponding author
}

\section{Article Info}

Keywords: Cubic B-spline collocation method, Finite difference method, Local discontinuous Galerkin method, Quenching time, Reaction-diffusion equation

2010 AMS: 65M06, 41A15, 65M60,

$35 K 57$

Received: 21 June 2020

Accepted: 05 November 2020

Available online: 15 December 2020

\begin{abstract}
In this paper, we study the numerical methods for solving a nonlinear reaction-diffusion model for the polarization phenomena in ionic conductors. In particular, we propose three types of numerical methods, including the finite difference, cubic B-spline collocation, and local discontinuous Galerkin method, to approximate the quenching time of the model. We prove the conservation properties for all three numerical methods and compare their numerical performance.
\end{abstract}

\section{Introduction}

In this paper, we investigate some numerical approximations of the quenching time of the solutions to the following nonlinear reaction-diffusion equation

$$
u_{t}(x, t)=u_{x x}(x, t)+\frac{1}{1-u(x, t)}, \quad 0<x<L, 0<t<T
$$

subject to the initial condition $u(x, 0)=0$ and two types of boundary conditions, i.e., the zero Neumann boundary condition

$$
u_{x}(0, t)=u_{x}(L, t)=0
$$

and the zero Dirichlet boundary condition

$$
u(0, t)=u(L, t)=0 .
$$

Here $L>0$ is a given constant. The quenching time of the solutions to the equation (1.1) with some initial and boundary conditions is defined to be the finite time $T$ (if it exists), such that

$$
\lim _{t \rightarrow T^{-}} \max \{u(x, t): 0 \leq x \leq L\} \rightarrow 1
$$

where $u(x, t)$ is the solution [1]. One can show that the initial-boundary value problem (1.1),(1.2) along with the zero initial condition leads to quenching at $T=1 / 2$. For the initial-boundary value problem $(1.1),(1.3)$ with the zero initial condition, 
it has been shown that the quenching time exists if $L>2 \sqrt{2}$ (see [2]). However, there is no analytical formulation for the quenching time. The objective of this paper is to propose numerical methods to approximate the quenching time, and prove their conservation property and compare their numerical performance. Specifically, we design the finite difference, cubic B-spline collocation and local discontinuous Galerkin method for equation (1.1) with Dirichlet and Neumann boundary conditions.

Finite difference methods have been widely used to solve a great number of differential equation models due to its simplicity. For certain nonlinear acoustics problems, however, it has been shown that the local discontinuous Galerkin (LDG) methods lead to more accurate solution than the benchmark finite difference methods in literature [3]. The LDG methods, as a special type of finite element methods, are suitable for complicated geometry and parallel computing. It is also easy to design LDG methods with high order of accuracy and conservation properties [4]-[6]. The cubic B-spline collocation methods have also been applied to solve various partial differential equations [7]. In addition, the Galerkin approach with the cubic B-spline has been applied to solve the modified regularized long wave equation [8], the generalized regularized long wave equation [9, 10], and the Benjamin-Bona-Mahony-Burgers equation [11]. One of the advantages of the cubic B-spline collocation methods over the finite element type of methods is that the calculation of coefficient matrices does not require numerical quadrature [12]. In this work, we propose three numerical methods based on the finite difference, cubic B-spline collocation and LDG methods. We can prove that all three numerical methods satisfy certain conservation property. We also compute the quenching time of equation (1.1) with Dirichlet and Neumann boundary conditions using these methods. To make fair comparison, we compute the results using three numerical methods which have the same order accuracy in both time and space.

The remaining of the paper is organized as follows. In section 2, we present the numerical methods for the nonlinear reactiondiffusion equation. In section 3 , we discuss the conservation properties of the proposed methods. In section 4 , we show the numerical results for the approximation of quenching time.

\section{Description of numerical methods}

In this section, we describe our numerical methods for solving the nonlinear reaction-diffusion equation (1.1) with zero initial condition, and Neumann boundary condition (1.2) or Dirichlet boundary condition (1.3). In particular, we focus on the finite difference method (FDM), the cubic B-splines method and the local discontinuous Galerkin (LDG) method, and investigate the performance of these numerical methods.

\subsection{Finite difference method}

We partition the spatial domain $[0, L]$ into $N$ elements uniformly using $(N+1)$ grid points: $0=x_{0}<x_{1}<\ldots<x_{N}=L$. We denote the length of each element by $h$, thus we have $h=L / N$ and $x_{i}=i h$ for $i=0,1, \ldots, N$. Suppose we want to solve the nonlinear reaction-diffusion equation (1.1) up to the final time $T$, then we discretize the temporal domain $[0, T]$ using $(M+1)$ uniformly spaced nodes $t_{j}:=j \Delta t$ for $j=0,1, \ldots, M$ with the step size $\Delta t=T / M$. Now we denote the numerical solution at $x=x_{i}$ and $t=t_{j}$ by $U_{i}^{j}$, then finite difference discretization of (1.1) can be written as

$$
\frac{U_{i}^{j+1}-U_{i}^{j}}{\Delta t}=\frac{U_{i-1}^{j}-2 U_{i}^{j}+U_{i+1}^{j}}{h^{2}}+\frac{1}{1-U_{i}^{j}},
$$

for $i=1,2, \ldots, N-1$ and $j=0,1, \ldots, M-1$. Here we have used the second-order centered difference in space and forward Euler method in time. That is,

$$
u_{t}\left(x_{i}, t_{j}\right) \approx \frac{U_{i}^{j+1}-U_{i}^{j}}{\Delta t}
$$

and

$$
u_{x x}\left(x_{i}, t_{j}\right) \approx \frac{U_{i-1}^{j}-2 U_{i}^{j}+U_{i+1}^{j}}{h^{2}}
$$

For the case of homogeneous Dirichlet boundary condition, we have

$$
U_{0}^{j}=U_{N}^{j}=0,
$$

for any $j$. Moreover, due to the initial condition, there is $U_{i}^{0}=0$ for any $i$. Therefore, at $(j+1)^{t h}$ time level $\left(j \leq M_{1}\right)$, we update $U_{i}^{j+1}$ by

$$
U_{i}^{j+1}=r U_{i+1}^{j}+(1-2 r) U_{i}^{j}+r U_{i-1}^{j}+\frac{\Delta t}{1-U_{i}^{j}}, \quad i=1,2, \ldots, N-1,
$$

and $U_{0}^{j+1}=U_{N}^{j+1}=0$. Here $r=\Delta t / h^{2}$ has to be chosen small enough for the stability of the scheme. 
For the case of homogeneous Neumann boundary condition, we introduce the ghost points $x_{-1}:=x_{0}-h$ and $x_{N+1}:=x_{N}+h$, and approximate the Neumann boundary condition using the second-order centered difference, which leads to

$$
U_{-1}^{j}=U_{1}^{j}, \quad U_{N+1}^{j}=U_{N-1}^{j} .
$$

Then we update $U_{0}^{j+1}$ and $U_{N}^{j+1}$ by applying (2.1) at $i=0$ and $i=N$, as well as conditions in (2.2). Therefore, the scheme for this case can be written as

$$
U_{0}^{j+1}=(1-2 r) U_{0}^{j}+2 r U_{1}^{j}+\frac{\Delta t}{1-U_{0}^{j}}, \quad U_{N}^{j+1}=2 r U_{N-1}^{j}+(1-2 r) U_{N}^{j}+\frac{\Delta t}{1-U_{N}^{j}},
$$

coupled with (2.1).

\subsection{Cubic B-spline collocation method}

The idea of the cubic B-spline collocation method is to look for the numerical solution represented using the cubic B-spline basis, such that equation (1.1) is satisfied at all the collocation points.

To describe the cubic B-spline collocation method, we use the same notation for the grid points in $[0, L]$ (see section 2.1), and define $x_{j}=j h$ for $j=0, \pm 1, \pm 2, \ldots$ Let $B_{j}(x)$ be the cubic B-spline function defined as

$$
B_{j}(x)=\frac{1}{h^{3}}\left\{\begin{array}{cc}
\left(x-x_{j-2}\right)^{3}, & \text { if } x \in\left[x_{j-2}, x_{j-1}\right) \\
\left(x-x_{j-2}\right)^{3}-4\left(x-x_{j-1}\right)^{3}, & \text { if } x \in\left[x_{j-1}, x_{j}\right) \\
\left(x_{j+2}-x\right)^{3}-4\left(x_{j+1}-x\right)^{3}, & \text { if } x \in\left[x_{j}, x_{j+1}\right) \\
\left(x_{j+2}-x\right)^{3}, & \text { if } x \in\left[x_{j+1}, x_{j+2}\right] \\
0, & \text { if } x \notin\left[x_{j-2}, x_{j+2}\right]
\end{array}\right.
$$

for any integer $j$. Note that each cubic B-spline basis function $B_{j}(x)$ is only nonzero when $x \in\left[x_{j-2}, x_{j+2}\right]$. Then the numerical solution $U(x, t)$ for $x \in[0, L]$ can be represented by

$$
U(x, t)=\sum_{j=-1}^{N+1} \alpha_{j}(t) B_{j}(x)
$$

since $B_{j}(x)=0$ for $x \in[0, L]$ and any $j \leq-2$ or $j \geq N+2$. Here $\alpha_{j}(t)$ for $j=-1,0, \ldots, N+1$ are the unknown coefficients to be determined.

For the case of homogeneous Dirichlet boundary condition, we require $U(x, t)$ in $(2.4)$ to satisfy equation $(1.1)$ at $x_{j}$, for $j=0,1, \ldots, N$, and vanish at $x=0$ and $x=L$. These conditions lead to $(N+3)$ ordinary differential equations with $(N+3)$ unknowns, which can be further solved by any time discretization method. With the help of the results in Table 1 , we can derive the formulation of the scheme as follows

$$
\alpha_{j-1}^{\prime}+4 \alpha_{j}^{\prime}+\alpha_{j+1}^{\prime}=\frac{6}{h^{2}}\left(\alpha_{j-1}-2 \alpha_{j}+\alpha_{j+1}\right)+\frac{1}{1-\alpha_{j-1}-4 \alpha_{j}-\alpha_{j+1}},
$$

for $j=0,1, \ldots, N$, coupled with

$$
\begin{aligned}
& U\left(x_{0}, t\right)=\alpha_{-1}+4 \alpha_{0}+\alpha_{1}=0, \\
& U\left(x_{N}, t\right)=\alpha_{N-1}+4 \alpha_{N}+\alpha_{N+1}=0 .
\end{aligned}
$$

If we apply (2.6) to (2.5) at $j=0$, then we can show that $\alpha_{-1}-2 \alpha_{0}+\alpha_{1}=-h^{2} / 6$, which leads to $\alpha_{0}=h^{2} / 36$ and $\alpha_{-1}=-h^{2} / 9-\alpha_{1}$. Similarly, if we apply (2.7) to (2.5), we can show that $\alpha_{N}=h^{2} / 36$ and $\alpha_{N+1}=-h^{2} / 9-\alpha_{N-1}$. If we further use these equations in (2.5), we can derive the following ODE system

$$
A \frac{d \alpha}{d t}=\mathbf{f}
$$

where

$$
A=\left[\begin{array}{cccccc}
4 & 1 & & & & \\
1 & 4 & 1 & & & \\
& 1 & 4 & 1 & & \\
& & \ddots & \ddots & \ddots & \\
& & & 1 & 4 & 1 \\
& & & & 1 & 4
\end{array}\right], \quad \alpha=\left[\begin{array}{c}
\alpha_{1} \\
\alpha_{2} \\
\vdots \\
\vdots \\
\alpha_{N-2} \\
\alpha_{N-1}
\end{array}\right] \quad \text { and } \quad \mathbf{f}=\left[\begin{array}{c}
f_{1} \\
f_{2} \\
\vdots \\
\vdots \\
f_{N-2} \\
f_{N-1}
\end{array}\right]
$$




\begin{tabular}{c|ccccc}
\hline & $x_{j-2}$ & $x_{j-1}$ & $x_{j}$ & $x_{j+1}$ & $x_{j+2}$ \\
\hline$B_{j}(x)$ & 0 & 1 & 4 & 1 & 0 \\
$B_{j}^{\prime}(x)$ & 0 & $3 / h$ & 0 & $-3 / h$ & 0 \\
$B_{j}^{\prime \prime}(x)$ & 0 & $6 / h^{2}$ & $-12 / h^{2}$ & $6 / h^{2}$ & 0 \\
\hline
\end{tabular}

Table 1: Evaluation of cubic B-spline basis function and its derivatives at grid points.

with $f_{j}=6\left(\alpha_{j-1}-2 \alpha_{j}+\alpha_{j+1}\right) / h^{2}+1 /\left(1-\alpha_{j-1}-4 \alpha_{j}-\alpha_{j+1}\right)$. We then solve equation (2.8) using the forward Euler method.

For the case of homogeneous Neumann boundary condition, we impose the boundary condition for $U(x, t)$ defined in $(2.4)$. Thus, we have

$$
\begin{array}{r}
U_{x}\left(x_{0}, t\right)=\frac{3}{h}\left(\alpha_{1}-\alpha_{-1}\right)=0 \\
U_{x}\left(x_{N}, t\right)=\frac{3}{h}\left(\alpha_{N+1}-\alpha_{N-1}\right)=0,
\end{array}
$$

which leads to $\alpha_{-1}=\alpha_{1}$ and $\alpha_{N+1}=\alpha_{N-1}$. We then apply these equations to (2.5) for $j=0,1, \ldots, N$, and obtain the following ODE system

$$
\tilde{A} \frac{d \tilde{\alpha}}{d t}=\tilde{\mathbf{f}}
$$

where

$$
\tilde{A}=\left[\begin{array}{cccccc}
4 & 2 & & & & \\
1 & 4 & 1 & & & \\
& 1 & 4 & 1 & & \\
& & \ddots & \ddots & \ddots & \\
& & & 1 & 4 & 1 \\
& & & & 2 & 4
\end{array}\right], \quad \tilde{\alpha}=\left[\begin{array}{c}
\alpha_{0} \\
\alpha_{1} \\
\vdots \\
\vdots \\
\alpha_{N-1} \\
\alpha_{N}
\end{array}\right] \quad \text { and } \quad \tilde{\mathbf{f}}=\left[\begin{array}{c}
f_{0} \\
f_{1} \\
\vdots \\
\vdots \\
f_{N-1} \\
f_{N}
\end{array}\right]
$$

Note that we can compute $f_{0}$ and $f_{N}$ using $f_{0}=12\left(\alpha_{1}-\alpha_{0}\right) / h^{2}+1 /\left(1-2 \alpha_{1}-4 \alpha_{0}\right)$ and $f_{N}=12\left(\alpha_{N-1}-\alpha_{N}\right) / h^{2}+1 /(1-$ $\left.2 \alpha_{N-1}-4 \alpha_{N}\right)$. Similarly, we can then solve equation (2.9) using the forward Euler method.

\subsection{Local discontinuous Galerkin method}

To define the local discontinuous Galerkin (LDG) method for the initial-boundary value problem, we use $I_{j}:=\left[x_{j-1}, x_{j}\right]$ to denote the $j^{\text {th }}$ element, and rewrite (1.1) as

$$
u_{t}=q_{x}+\frac{1}{1-u}, \quad q=u_{x}
$$

Let $V_{h}^{k}$ be the piecewise defined polynomial space given by $V_{h}^{k}=\left\{v:\left.v\right|_{I_{j}} \in P^{k}\left(I_{j}\right), \forall I_{j}\right\}$, where $P^{k}\left(I_{j}\right)$ is the space of polynomials of degree up to $k$ on $I_{j}$. The LDG scheme is to look for $u_{h}$ and $q_{h} \in V_{h}^{k}$, such that

$$
\begin{aligned}
\int_{I_{j}} \frac{\partial}{\partial t} u_{h} v d x & =-\int_{I_{j}} q_{h} v_{x} d x+\widehat{q}_{j} v_{j}^{-}-\widehat{q}_{j-1} v_{j-1}^{+}+\int_{I_{j}} \frac{v}{1-u_{h}} d x \\
\int_{I_{j}} q_{h} w d x & =-\int_{I_{j}} u_{h} w_{x} d x+\widehat{u}_{j} w_{j}^{-}-\widehat{u}_{j-1} w_{j-1}^{+}
\end{aligned}
$$

for any $v, w \in V_{h}^{k}$ and $j=1,2, \ldots, N$. Here $v_{j}^{-}:=\lim _{\varepsilon \rightarrow 0+} v\left(x_{j}-\varepsilon\right)$ and $v_{j-1}^{+}:=\lim _{\varepsilon \rightarrow 0+} v\left(x_{j-1}+\varepsilon\right)$ are the left- and right-hand limit of $v(x)$ at $x_{j+\frac{1}{2}}$ and $x_{j-\frac{1}{2}}$, respectively. Similar definition holds for $w_{j}^{-}$and $w_{j-1}^{+}$. Due to the discontinuity of the numerical solution at the grid points, we use $\widehat{q}_{j}$ and $\widehat{u}_{j}$ for $j=0,1, \ldots, N$, to denote the so-called numerical fluxes, which will be carefully chosen for different boundary conditions.

For the initial-boundary value problem with the homogeneous Dirichlet boundary condition, we choose

$$
\widehat{q}_{j}=\left(q_{h}\right)_{j}^{+}, \quad \widehat{u}_{j}=\left(u_{h}\right)_{j}^{-}, \quad \text { for } j=1,2, \ldots, N-1 .
$$


That is, at any interior grid points $x_{j}$, the numerical fluxes $\widehat{q}_{j}$ and $\widehat{u}_{j}$ are computed using $\left.q_{h}\right|_{I_{j+1}}$ (i.e., the restriction of the function $q_{h}$ on the interval $I_{j+1}$ ) and $\left.u_{h}\right|_{I_{j}}$ (i.e., the restriction of the function $u_{h}$ on the interval $I_{j}$ ), respectively. At the left and right endpoints, we take

$$
\widehat{q}_{0}=\left(q_{h}\right)_{0}^{+}, \quad \widehat{u}_{0}=0, \quad \widehat{q}_{N}=\left(q_{h}\right)_{N}^{-}-\frac{1}{h}\left(u_{h}\right)_{N}^{-}, \quad \widehat{u}_{N}=0
$$

Note that we have used the penalty term $\left(u_{h}\right)_{N}^{-} / h$ in the definition of $\widehat{q}_{N}$. Such a term is crucial when we design the numerical scheme [13].

For the problem with the homogeneous Neumann boundary condition, we use (2.12) along with the following choice at the boundary [14]:

$$
\widehat{q}_{0}=0, \quad \widehat{u}_{0}=\left(u_{h}\right)_{0}^{+}, \quad \widehat{q}_{N}=0, \quad \widehat{u}_{N}=\left(u_{h}\right)_{N}^{-} .
$$

Let $\left\{v_{1}^{j}(x), v_{2}^{j}(x), \ldots, v_{k+1}^{j}(x)\right\}$ be a local basis of $V_{h}^{k}$ on $I_{j}(1 \leq j \leq N)$. We can express the numerical solution on $I_{j}$ as

$$
\left.u_{h}\right|_{I_{j}}=\sum_{l=1}^{k+1} C_{l}^{j}(t) v_{l}^{j}(x),\left.\quad q_{h}\right|_{I_{j}}=\sum_{l=1}^{k+1} D_{l}^{j}(t) v_{l}^{j}(x)
$$

where $\left\{C_{l}^{j}(t)\right\}_{l=1, \ldots, k+1}$ and $\left\{D_{l}^{j}(t)\right\}_{l=1, \ldots, k+1}$ are the unknown coefficients to be determined. If we use (2.15) in equations (2.10)-(2.13) (for the case of Dirichlet boundary condition), or equations (2.10)-(2.12), (2.14) (for the case of Neumann boundary condition), we can derive an ODE system of $C_{l}^{j}(t)$ and $D_{l}^{j}(t)$ for $l=1,2, \ldots, k+1$ and $j=1,2, \ldots, N$, which will be solved by any time integration method.

\section{Conservation properties of numerical methods}

In this section, we present some properties of the three numerical methods described in section 2, when they are used to solve the initial-boundary value problem (1.1) with zero initial condition and the boundary condition (1.2). Throughout this section, we assume that the quenching time of such a problem is $T>0$. Then it is easy to verify that its exact solution $u(x, t)$ satisfies the following equalities:

$$
\int_{0}^{L} u_{t} d x=\int_{0}^{L} \frac{1}{1-u} d x
$$

and

$$
\frac{1}{2} \frac{d}{d t} \int_{0}^{L} u^{2} d x+\int_{0}^{L} u_{x}^{2} d x=\int_{0}^{L} \frac{u}{1-u} d x
$$

for any $t<T$. We will show that similar equalities also hold for the numerical solutions.

Theorem 3.1. The numerical solution by the finite difference method in equation (2.1) and (2.3) satisfies the following equation

$$
h \sum_{i=0}^{N} d_{i} U_{i}^{j+1}=h \sum_{i=0}^{N} d_{i} U_{i}^{j}+h \Delta t \sum_{i=0}^{N} \frac{d_{i}}{1-U_{i}^{j}}
$$

if $U_{i}^{j}<1$ for $i=0, \ldots, N$. Here $d_{i}=1$ for $i=0$ or $N$, and $d_{i}=2$ for $i=1,2, \ldots, N-1$.

Proof. We multiply each equation in (2.1) by 2 and sum over $i$ for $i=1,2, \ldots, N-1$, to get

$$
\begin{aligned}
\sum_{i=1}^{N-1} d_{i} U_{i}^{j+1} & =\sum_{i=1}^{N-1} d_{i} U_{i}^{j}+\sum_{i=1}^{N-1} 2 r U_{i-1}^{j}+\sum_{i=1}^{N-1} 2 r U_{i+1}^{j}-\sum_{i=1}^{N-1} 4 r U_{i}^{j}+\Delta t \sum_{i=1}^{N-1} \frac{d_{i}}{1-U_{i}^{j}} \\
& =\sum_{i=1}^{N-1} d_{i} U_{i}^{j}+2 r U_{0}^{j}-2 r U_{1}^{j}+2 r U_{N}^{j}-2 r U_{N-1}^{j}+\Delta t \sum_{i=1}^{N-1} \frac{d_{i}}{1-U_{i}^{j}} .
\end{aligned}
$$

We then add each of the equations in (2.3) to equation (3.4), and obtain

$$
U_{0}^{j+1}+U_{N}^{j+1}+\sum_{i=1}^{N-1} d_{i} U_{i}^{j+1}=U_{0}^{j}+U_{N}^{j}+\sum_{i=1}^{N-1} d_{i} U_{i}^{j}+\Delta t \sum_{i=1}^{N} \frac{d_{i}}{1-U_{i}^{j}} .
$$

Since equation (3.5) is equivalent to (3.3), we can conclude the proof. 
Note that $h \sum_{i=0}^{N} d_{i} U_{i}^{j+1}$ is the approximation of $\int_{0}^{L} u\left(x, t_{j+1}\right) d x$ using the trapezoidal rule, and the right side of (3.3) is the approximation of $\int_{0}^{L} u\left(x, t_{j}\right) d x+\int_{0}^{L} \frac{1}{u\left(x, t_{j}\right)} d x$. Therefore, we can regard equation (3.3) as the discretization of the equality (3.1). Next, we consider the cubic B-spline method in (2.9) and we have the following theorem.

Theorem 3.2. With the cubic B-spline method defined in (2.9), the following equality holds

$$
6 h \sum_{i=0}^{N} d_{i} \alpha_{i}^{\prime}(t)=\frac{h}{1-4 \alpha_{0}-2 \alpha_{1}}+\sum_{i=1}^{N-1} \frac{2 h}{1-\alpha_{i-1}-4 \alpha_{i}-\alpha_{i+1}}+\frac{h}{1-2 \alpha_{N-1}-4 \alpha_{N}}
$$

if $4 \alpha_{0}+2 \alpha_{1}<1,2 \alpha_{N-1}+4 \alpha_{N}<1$ and $\alpha_{i-1}+4 \alpha_{i}+\alpha_{i+1}<1$ for $i=1,2, \ldots, N-1$.

Proof. Equation (2.9) leads to

$$
\begin{aligned}
4 \alpha_{0}^{\prime}+2 \alpha_{1}^{\prime} & =\frac{12}{h^{2}}\left(\alpha_{1}-\alpha_{0}\right)+\frac{1}{1-4 \alpha_{0}-2 \alpha_{1}} \\
\alpha_{j-1}^{\prime}+4 \alpha_{j}^{\prime}+\alpha_{j+1}^{\prime} & =\frac{6}{h^{2}}\left(\alpha_{j-1}-2 \alpha_{j}+\alpha_{j+1}\right)+\frac{1}{1-\alpha_{j-1}-4 \alpha_{j}-\alpha_{j+1}} \\
2 \alpha_{N-1}^{\prime}+4 \alpha_{N}^{\prime} & =\frac{12}{h^{2}}\left(\alpha_{N-1}-\alpha_{N}\right)+\frac{1}{1-2 \alpha_{N-1}-4 \alpha_{N}}
\end{aligned}
$$

for $j=1,2, \ldots, N-1$. We then multiply each equation in (3.8) by $2 h$, sum over $j$ and add (3.7) and (3.9), which leads to $(3.6)$.

Since $\int_{0}^{L} u_{t} d x \approx h \sum_{i=0}^{N} d_{i} U_{t}\left(x_{i}, t\right)=h \sum_{i=0}^{N} d_{i}\left(\alpha_{i-1}^{\prime}+4 \alpha_{i}^{\prime}+\alpha_{i+1}^{\prime}\right)=6 h \sum_{i=0}^{N} d_{i} \alpha_{i}^{\prime}$, and the right side of (3.6) is an approximation of $\int_{0}^{L} \frac{1}{1-u} d x$, equation (3.6) is also the discretization of the equality (3.1). The next theorem is about the conservation property of the local discontinuous Galerkin method (2.10), (2.11), (2.12) and (2.14).

Theorem 3.3. Let $u_{h}$ and $q_{h}$ be the numerical solution to (2.10), (2.11), (2.12) and (2.14), then the following equality holds for sufficiently small $t>0$

$$
\frac{1}{2} \frac{d}{d t} \int_{0}^{L} u_{h}^{2} d x+\int_{0}^{L} q_{h}^{2} d x=\int_{0}^{L} \frac{u_{h}}{1-u_{h}} d x
$$

Proof. Let $v=u_{h}$ in (2.10), $w=q_{h}$ in (2.11) and add the resulting equations, we have

$$
\begin{aligned}
& \frac{1}{2} \frac{d}{d t} \int_{I_{j}} u_{h}^{2} d x+\int_{I_{j}} q_{h}^{2} d x \\
= & -\int_{I_{j}}\left(q_{h} u_{h}\right)_{x} d x+\widehat{q}_{j}\left(u_{h}\right)_{j}^{-}-\widehat{q}_{j-1}\left(u_{h}\right)_{j-1}^{+}+\widehat{u}_{j}\left(q_{h}\right)_{j}^{-}-\widehat{u}_{j-1}\left(q_{h}\right)_{j-1}^{+}+\int_{I_{j}} \frac{u_{h}}{1-u_{h}} d x \\
= & \left(\left(q_{h}\right)_{j-1}^{+}-\widehat{q}_{j-1}\right)\left(u_{h}\right)_{j-1}^{+}+\left(\widehat{u}_{j}-\left(u_{h}\right)_{j}^{-}\right)\left(q_{h}\right)_{j}^{-}+\widehat{q}_{j}\left(u_{h}\right)_{j}^{-}-\widehat{u}_{j-1}\left(q_{h}\right)_{j-1}^{+}+\int_{I_{j}} \frac{u_{h}}{1-u_{h}} d x,
\end{aligned}
$$

for $j=1,2, \ldots, N$. Using equation (2.12), we can show that equation (3.11) leads to

$$
\frac{1}{2} \frac{d}{d t} \int_{I_{j}} u_{h}^{2} d x+\int_{I_{j}} q_{h}^{2} d x=\left(q_{h}\right)_{j}^{+}\left(u_{h}\right)_{j}^{-}-\left(q_{h}\right)_{j-1}^{+}\left(u_{h}\right)_{j-1}^{-}+\int_{I_{j}} \frac{u_{h}}{1-u_{h}} d x
$$

for any $j=2,3, \ldots, N-1$. For $j=1$, equation (3.11) can be simplified as

$$
\begin{aligned}
\frac{1}{2} \frac{d}{d t} \int_{I_{1}} u_{h}^{2} d x+\int_{I_{1}} q_{h}^{2} d x & =\left(\left(q_{h}\right)_{0}^{+}-\widehat{q}_{0}\right)\left(u_{h}\right)_{0}^{+}+\left(q_{h}\right)_{1}^{+}\left(u_{h}\right)_{1}^{-}-\widehat{u}_{0}\left(q_{h}\right)_{0}^{+}+\int_{I_{1}} \frac{u_{h}}{1-u_{h}} d x \\
& =\left(q_{h}\right)_{1}^{+}\left(u_{h}\right)_{1}^{-}+\int_{I_{1}} \frac{u_{h}}{1-u_{h}} d x
\end{aligned}
$$

where we have used (2.12) and (2.14) in the first and second equality above, respectively. For $j=N$, equation (3.11) can be written as

$$
\begin{aligned}
& \frac{1}{2} \frac{d}{d t} \int_{I_{N}} u_{h}^{2} d x+\int_{I_{N}} q_{h}^{2} d x \\
= & \left(\left(q_{h}\right)_{N-1}^{+}-\widehat{q}_{N-1}\right)\left(u_{h}\right)_{N-1}^{+}+\left(\widehat{u}_{N}-\left(u_{h}\right)_{N}^{-}\right)\left(q_{h}\right)_{N}^{-}+\widehat{q}_{N}\left(u_{h}\right)_{N}^{-}-\widehat{u}_{N-1}\left(q_{h}\right)_{N-1}^{+}+\int_{I_{N}} \frac{u_{h}}{1-u_{h}} d x, \\
= & -\left(u_{h}\right)_{N-1}^{-}\left(q_{h}\right)_{N-1}^{+}+\int_{I_{N}} \frac{u_{h}}{1-u_{h}} d x .
\end{aligned}
$$


Finally, we add (3.12)-(3.14) over all $j=2,3, \ldots, N-1$ to get

$$
\begin{aligned}
& \frac{1}{2} \frac{d}{d t} \int_{0}^{L} u_{h}^{2} d x+\int_{0}^{L} q_{h}^{2} d x \\
= & \sum_{j=2}^{N-1}\left(\left(q_{h}\right)_{j}^{+}\left(u_{h}\right)_{j}^{-}-\left(q_{h}\right)_{j-1}^{+}\left(u_{h}\right)_{j-1}^{-}\right)+\left(q_{h}\right)_{1}^{+}\left(u_{h}\right)_{1}^{-}-\left(u_{h}\right)_{N-1}^{-}\left(q_{h}\right)_{N-1}^{+}+\int_{0}^{L} \frac{u_{h}}{1-u_{h}} d x \\
= & \int_{0}^{L} \frac{u_{h}}{1-u_{h}} d x
\end{aligned}
$$

Note that equation (3.10) is the discretized version of the conservation property (3.2) in the PDE level.

\section{Numerical experiments}

In this section, we present the numerical results of the quenching time for the nonlinear reaction-diffusion equation (1.1). We compare the accuracy of the finite difference method, the cubic B-spline collocation method and the local discontinuous Galerkin method for the quenching time. We choose the polynomial degree to be $k=1$ for the LDG method, so that all three numerical methods have the second-order accuracy in space. Numerically, we compute the quenching time $T$ in the following way

$$
T:=\min _{t}\left(\max _{x \in[0, L]} U(x, t)\right) \geq 1-\varepsilon,
$$

where $U(x, t)$ is the numerical solution, and $\varepsilon$ is chosen to be $10^{-7}$.

We first consider equation (1.1) with zero initial condition and the homogeneous Neumann boundary condition, in which case the exact solution is

$$
u(x, t)=1-\sqrt{1-2 t}, \quad t \leq 1 / 2 .
$$

Thus the exact quenching time is $t=1 / 2$. We then use the above-mentioned numerical methods to approximate the quenching time. The results for the case of Neumann boundary condition are shown in Table 2 . We have used $\Delta t=\lambda h^{2}$ with $\lambda=0.16$ in all the simulations for the table. We start with the numerical simulations using $N=80$ uniform elements, and then double the number of elements a few times until $N=640$. Suppose the errors corresponding to $N_{1}$ and $N_{2}:=N_{1} / 2$ are $E_{1}$ and $E_{2}$, respectively. We can approximate the convergence order by

$$
\text { Convergence order } \approx \log _{2}\left(E_{2} / E_{1}\right) .
$$

From Table 2, we observe the second order convergence of the quenching time for all three numerical methods. Moreover, we find that the numerical quenching time of the local discontinuous Galerkin method is exactly the same as that of the finite difference method. However, the results of the cubic B-spline method is less accurate then other two methods. Overall speaking, all of the methods lead to the numerical quenching time with comparable accuracy. Figure 4.1 shows the numerical solutions at various time, i.e., $t=0.1,0.2, \ldots, 0.5$, when the finite difference method with 80 elements is used. We can see that the method captures the quenching phenomena, although the numerical solution at $t=0.5$ is slightly smaller than the exact solution.

\begin{tabular}{|c|ccc|ccc|ccc|}
\hline & \multicolumn{4}{|c|}{ Finite Difference } & \multicolumn{3}{c|}{ Cubic B-Spline } & \multicolumn{3}{c|}{ Local Discontinuous Galerkin } \\
\hline$N$ & Time & Error & Order & Time & Error & Order & Time & Error & Order \\
80 & 0.50120 & $1.2 \mathrm{E}-3$ & - & 0.50200 & $2.0 \mathrm{E}-3$ & - & 0.50120 & $1.2 \mathrm{E}-3$ & - \\
160 & 0.50030 & $3.0 \mathrm{E}-4$ & 2 & 0.50050 & $5.0 \mathrm{E}-4$ & 2 & 0.50030 & $3.0 \mathrm{E}-4$ & 2 \\
320 & 0.50010 & $1.0 \mathrm{E}-4$ & 1.585 & 0.50015 & $1.5 \mathrm{E}-4$ & 1.737 & 0.50010 & $1.0 \mathrm{E}-4$ & 1.585 \\
640 & 0.50003 & $2.5 \mathrm{E}-5$ & 2 & 0.50004 & $3.75 \mathrm{E}-5$ & 2 & 0.50003 & $2.5 \mathrm{E}-5$ & 2 \\
\hline
\end{tabular}

Table 2: Numerical results about the quenching time of equation (1.1) with homogeneous Neumann boundary condition.

Next, we consider equation (1.1) with zero initial condition and the homogeneous Dirichlet boundary condition. In [2], the author proved that this problem leads to quenching in a finite time at $x=L / 2$. However, the analytical formulation or any estimation of the quenching time was not discussed. Here we compute the quenching time using the aforementioned numerical methods. In this case, we start with $N=80$ elements and keep refining the mesh until $N=2560$ elements. Figure 4.2 shows the solutions by the finite difference method with $N=80$ elements. We observe that the numerical solution at $x=L / 2$ grows at an increasing rate. When we compare the numerical results in Figure 4.1 and 4.2, we notice that the problem with the Dirchlet boundary condition (1.3) leads to larger quenching time than the problem with the Neumann boundary condition (1.2). The numerical solution $u_{h}(x, t)$ and $q_{h}(x, t)$ by the LDG method right before quenching occurs is shown in Figure 4.3. We observe 


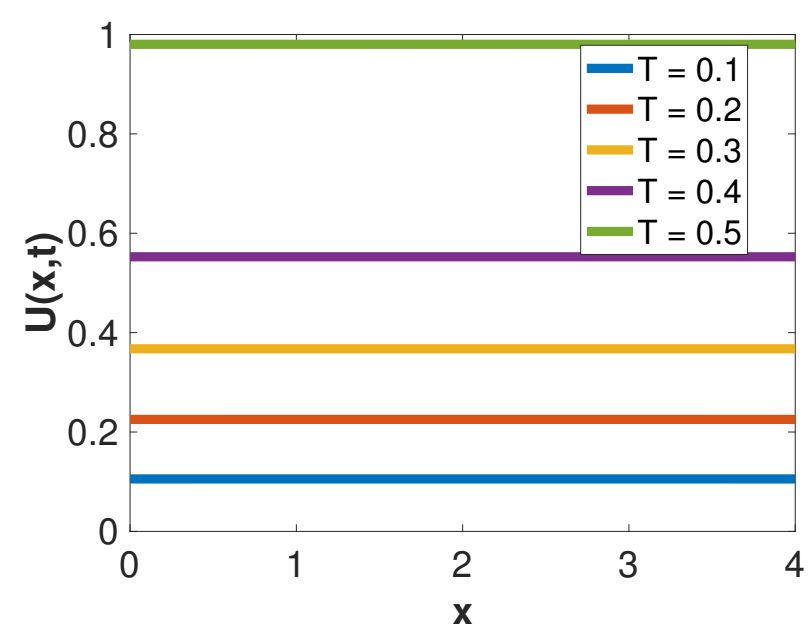

Figure 4.1: Solutions of equation (1.1) with homogeneous Neumann boundary condition by the finite difference method. $N=80$ is used for the simulation.

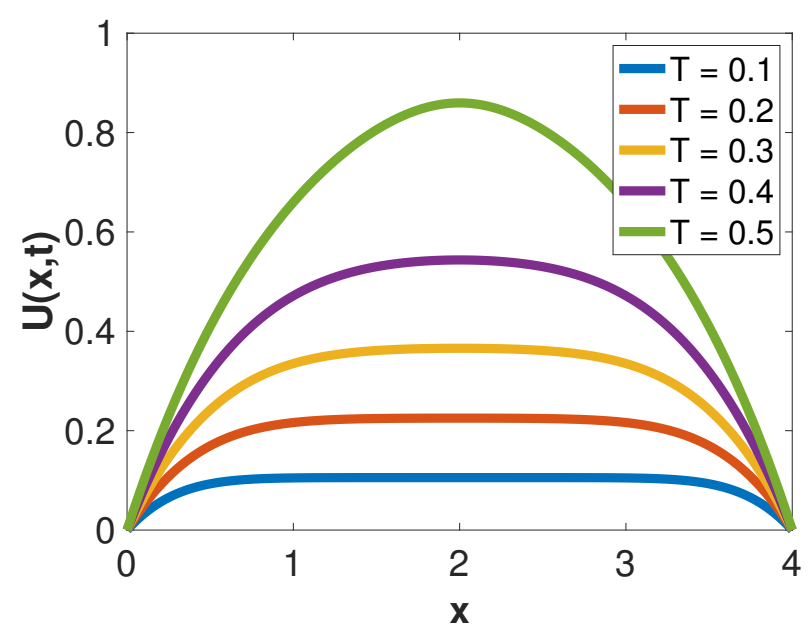

Figure 4.2: Solutions of equation (1.1) with homogeneous Dirichlet boundary condition by the finite difference method. $N=80$ is used for the simulation.

that $u_{h}$ is not very smooth at $x=2$, and the quenching is about to happen at this point. Recall that $q_{h}$ is the approximation of first derivative of the exact solution $u$. The right-hand figure 4.3 shows that the first derivative of the solution decreases, and jumps to a negative value at $x \rightarrow 2^{+}$.

Moreover, we compute the numerical quenching time for different numerical methods, and present the results in Table 3 . We can see that the quenching time decreases monotonically at a decreasing rate for the finite difference and LDG methods, which indicates the convergence. However, the quenching time for the cubic B-spline method first decreases to 0.51035 and then increases to 0.51039 . The results from the LDG method shows similar type of convergence as that from the finite difference method, i.e., the numerical quenching time converges to 0.51041 for both methods. Overall speaking, for both examples with the same number of elements, the LDG and finite difference method lead to slightly better results then the cubic B-spline method.

\section{Conclusion}

In this paper, we propose the finite difference, the cubic B-spline and the local discontinuous Galerkin methods to approximate the quenching time of a nonlinear reaction-diffusion equation with homogeneous Dirichlet or Neumann boundary condition. All of these numerical methods have displayed satisfactory results, with the second order convergence of the numerical quenching time. For the same number of elements in space, the finite difference and the local discontinuous Galerkin methods have slightly better performance than the cubic B-spline method. One can propose similar methods for higher dimensional system of reaction-diffusion equations. The theoretical analysis for the convergence of the numerical quenching time is currently under investigation. 

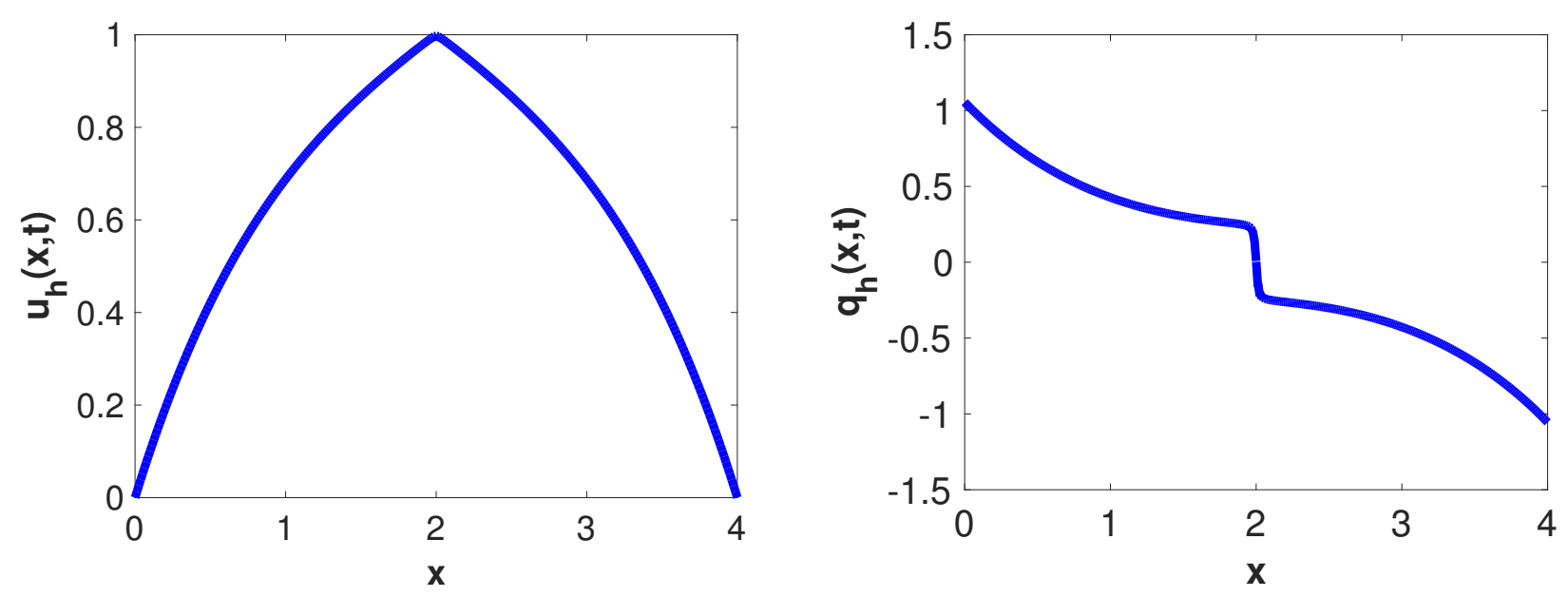

Figure 4.3: Numerical solution $u_{h}$ and $q_{h}$ of equation (1.1) with homogeneous Dirichlet boundary condition right before quenching occurs. Left: numerical solution $u_{h}$; right: numerical solution $q_{h}$ The local discontinuous Galerkin method with $N=320$ is used for the simulation.

\begin{tabular}{|c|c|c|c|}
\hline $\mathrm{N}$ & Finite Difference & Cubic B-Spline & Local Discontinuous Galerkin \\
\hline 80 & 0.52250 & 0.51160 & 0.51070 \\
160 & 0.51080 & 0.51050 & 0.51050 \\
320 & 0.51053 & 0.51035 & 0.51043 \\
640 & 0.51044 & 0.51035 & 0.51042 \\
1280 & 0.51042 & 0.51037 & 0.51041 \\
2560 & 0.51041 & 0.51039 & 0.51041 \\
\hline
\end{tabular}

Table 3: Numerical results about the quenching time of equation (1.1) with Dirichlet boundary condition (1.3).

\section{References}

[1] B. Selcuk, N. Ozalp, Quenching behavior of semilinear heat equations with singular boundary conditions, Electron. J. Differ. Eq., 311 (2015), 1-13.

[2] H. Kawarada, On solutions of initial-boundary problem for $u_{t}=u_{x x}+\frac{1}{1-u}$, Publ. RIMS, Kyoto Univ., 10 (1975), 729-736.

[3] C. S. Chou, W. Sun, Y. Xing, H. Yang, Local discontinuous Galerkin methods for the Khokhlov-Zabolotskaya-Kuznetzov equation, J. Sci. Comput., 73 (2017), 593-616.

[4] H. Yang, High-order energy and linear momentum conserving methods for the Klein-Gordon equation, Math., (2018), Article ID 200,17 pages.

[5] H. Yang, Error estimates for a class of energy-and Hamiltonian-preserving local discontinuous Galerkin methods for the Klein-Gordon-Schrödinger equations, J. Appl. Math. Comput., 62 (2020), 377-424.

[6] H. Yang, Optimal error estimate of a decoupled conservative local discontinuous Galerkin method for the Klein-Gordon-Schrödinger equations, J. Korean Soc. Ind. Appl. Math., 24 (2020), 39-78.

[7] R. C. Mittal, R. K. Jain, Cubic B-splines collocation method for solving nonlinear parabolic partial differential equations with Neumann boundary conditions, Commun. Nonlinear. Sci., 17 (2012), 4616-4625.

[8] S. B. G. Karakoç, Y. Uçar, N. Yagmurluğ, Numerical solutions of the MRLW equation by cubic B-spline Galerkin finite element method, Kuwait J. Sci., 42 (2015), 141-159.

[9] H. Zeybek, S. B. G. Karakoç, A numerical investigation of the GRLW equation using lumped Galerkin approach with cubic B-spline, SpringerPlus, (2016), Article ID 199, 17 pages.

[10] S. K. Bhowmik, S.B.G. Karakoç, Numerical approximation of the generalized regularized long wave equation using Petrov-Galerkin finite element method, Numer. Methods Partial Differ. Equ., 35 (2019), 2236-2257.

[11] S. B. G. Karakoç, S. K. Bhowmik, Galerkin finite element solution for Benjamin-Bona-Mahony-Burgers equation with cubic B-splines, Comput. Math. Appl., 77 (2019), 1917-1932.

[12] X. Ding, Q. J. Meng, L. P. Yin, Discrete-time orthogonal spline collocation method for one-dimensional sine-Gordon equation, Discrete Dyn. Nat. Soc., (2015), Article ID 206264, 8 pages.

[13] L. Guo, Y. Yang, Positivity preserving high-order local discontinuous Galerkin method for parabolic equations with blow-up solutions, J. Comput. Phys., 289 (2015), 181-195.

[14] L. Shao, X. Feng, Y. He, The local discontinuous Galerkin finite element method for Burger's equation, Math. Comput. Model., 54 (2011), $2943-2954$. 